\title{
MHD Equilibria of Tokamak Plasmas
}

\author{
Carles Barril $^{1}$, Alex Haro ${ }^{2}$, Marta Pellicer ${ }^{3} \dagger$ and Joan Solà-Morales ${ }^{4}$ \\ ${ }^{1}$ Universitat Autònoma de Barcelona, Spain \\ ${ }^{2}$ Universitat de Barcelona, Spain \\ ${ }^{3}$ Universitat de Girona, Spain \\ ${ }^{4}$ Universitat Politècnica de Catalunya, Spain \\ (Communicated to MIIR on 26 April 2021)
}

Study Group: ESGI 115. 25-29 January, 2016, Barcelona, Spain

Communicated by: Tim Myers

Industrial Partner: Fusion for Energy, Barcelona, Spain

Presenter: Alfredo Portone

Team Members: Carles Barril, Barcelona; Alex Haro, Barcelona; Jesús Fernández-Sánchez, Barcelona; Giorgi Khimshiashvili, Tbilisi; Marta Pellicer, Girona; Joan Solà-Morales, Barcelona.

Industrial Sector: Energy/Utilities

Tools: Level Curve, Polynomial Approximation, Plasma Physics

Key Words: Tokamak, Plasmas, Fusion

MSC2020 Codes: 76

\section{Summary}

The solution of the Grad-Shafranov (GS) equation, which is at the basis of Tokamak plasma MHD equilibrium computation, in the fixed boundary formulation first requires the description of the plasma boundary in terms of a few physical quantities. Limiter plasmas are characterized by a boundary that is a closed magnetic surface tangent to a material surface for which there is a simple parametrization in terms of those quantities. Divertor plasmas are, by contrast, characterized by a boundary that is not a closed flux surface nor tangent to any material surface, but contains saddle point(s) at which the boundary has cusp(s) of $90^{\circ}$. For such a boundary the limiter plasma parametrization is no longer valid. We investigate whether there is an analogous simple parametrization of divertor plasma boundaries.

$\dagger$ Corresponding Author: marta.pellicer@udg.edu 


\title{
MHD Equilibria of Tokamak Plasmas
}

\author{
Problem presented by
}

Alfredo Portone ${ }^{1}$

Report prepared by

Carles Barril $^{2}$, Àlex Haro ${ }^{3}$, Marta Pellicer ${ }^{4,7}$, Joan Solà-Morales ${ }^{5}$

Study group contributors

Carles Barril $^{2}$, Àlex Haro ${ }^{3}$, Jesús Fernández-Sánchez ${ }^{5}$, Giorgi Khimshiashvili ${ }^{6}$, Marta Pellicer $^{4,7}$, Joan Solà-Morales ${ }^{5}$

\section{Statement of the problems}

The ITER project is an international project being developed in southern France. There, a world-wide consortium (China, the European Union, India, Japan, Korea, Russia and the United States) has launched the ITER initiative to design and build the world's largest tokamak, an experimental magnetic fusion device that has been developed to prove the feasibility of nuclear fusion as a large-scale and carbon-free source of energy. A tokamak is a device that confines a plasma by means of a toroidal magnetic field generated by external coils (see http://en.wikipedia.org/wiki/Tokamak). Actually, the term "tokamak", of Russian origin, stands for "toroidal chamber with magnetic coils". For stable equilibria, azimuthal (i.e., rotational) symmetry is required.

Fusion for Energy (F4E) is the European Union's Joint Undertaking for ITER and the Development of Fusion Energy. F4E is responsible, among others, for providing Europe's contribution to ITER project. This European Agency suggested the following two different problems to be discussed at the 115th European Study Group with Industry.

\subsection{Problem 1: plasma boundary parametrization}

The solution of the Grad-Shafranov equation [4], which is at the basis of tokamak plasma MHD equilibrium computations, in the fixed boundary formulation first requires the de-

\footnotetext{
${ }^{1}$ Fusion for Energy, Barcelona, Spain

${ }^{2}$ Universitat Autònoma de Barcelona, Bellaterra, Spain

${ }^{3}$ Universitat de Barcelona, Barcelona, Spain

${ }^{4}$ Universitat de Girona, Girona, Spain

${ }^{5}$ Universitat Politècnica de Catalunya, Barcelona, Spain

${ }^{6}$ Ilia State University, Tbilisi, Georgia

${ }^{7}$ Report co-ordinator: marta.pellicer@udg.edu
} 
scription of the plasma boundary in terms of a few geometrical quantities. Typically, such quantities are $R, a, k$ and $\delta$, that is, the major and minor radius, the elongation and the triangularity of the toroidal plasma.

Limiter plasmas are characterized by a boundary that is a closed magnetic surface that is tangent to a material surface (poloidal or toroidal limiter). In cylindrical coordinates $(r, \varphi, z)$, the flux does not depend on the $\varphi$ angle (is axisymmetric). A common description for such type of boundary in the $(r, z)$ section is given by the following parametrization (see [2] for details):

$$
\left\{\begin{array}{l}
r(\theta)=R+a \cos (\theta+\delta \sin (\theta)) \\
z(\theta)=k \sin (\theta)
\end{array}\right.
$$

where $\theta \in[0,2 \pi)$ represents the poloidal angle between the $r$-axis and the line connecting $(R, 0)$ and $(r, z)$ (the generic plasma boundary point in the poloidal plane).

Divertor plasmas are characterized by a boundary that is not in contact with any material surface and contains a saddle point at which the boundary has a corner of 90 degrees. For such type of boundary (which is the most commonly used in burning plasmas) the parametrization above is no longer valid.

The Fusion for Energy (F4E) problem consists in finding a suitable family of curves (and depending on control parameters) whose only constraints in the $(r, z)$ plane are:

(1) The curve must be tangent parallel to the $z$-axis (that is, vertical direction) at $\left(R_{i}, 0\right)$ and $\left(R_{o}, 0\right)$, the points of minimum and maximum radii of the curve, respectively. $R_{i}$ and $R_{o}$ should be specified by the user.

(2) The curve must contain a saddle point at $\left(R_{x}, Z_{x}\right)$ where the branches cross at 90 degrees. $\left(R_{x}, Z_{x}\right)$ should be specified by the user.

(3) The curve is not necessarily symmetric with respect to $z=0$.

The curve should be given in terms of some (easy) geometric quantities.

\subsection{Problem 2: plasma boundary control}

The boundary of a tokamak plasma is controlled by acting on the currents flowing in the surrounding Poloidal Field (PF) coils. By solving the fixed boundary Grad-Shafranov equilibrium problem (see above) we easily establish the target flux function $\Psi$ along the target plasma boundary $\Gamma$ that must be matched by the currents flowing in the external $\mathrm{PF}$ coils. A typical example of such functions for different type of equilibria is shown in the figure below.

Perfect matching is possible only if the set of PF currents are placed along the plasma boundary itself [4], which is technically impossible. A common problem in tokamak engineering is therefore, to find a set of PF currents located on a different contour $\Gamma^{*}$, external to $\Gamma$, that match at best such target flux function $\Psi$ while minimizing a weighted functional of error (JE) and cost (JC) (typically expressed in terms of magnetic energy, see $[3,4])$.

The problem consists in providing an algorithm to compute the PF centers location and current along $\Gamma^{*}$ to optimize a given functional $\mathrm{J}=\mathrm{JE}+\mathrm{JC}$. 


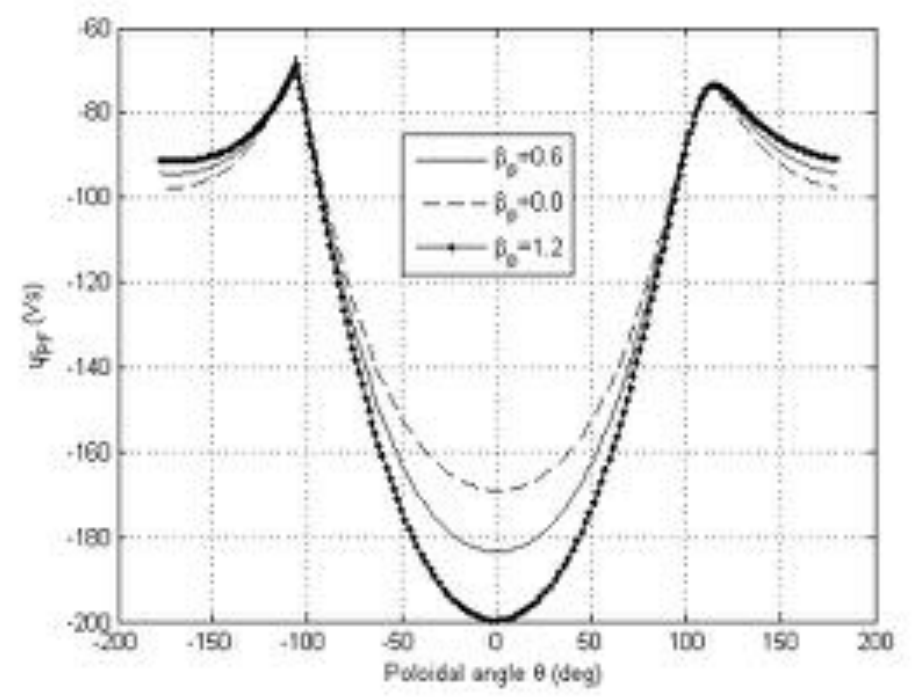

\section{Problem 1: general remarks}

We have proceeded to solve this problem mainly by two methods, that will be described in Sections 2 and 3 below. Before going into that, we give some general remarks. Without loss of generality, we assume a coordinate reference system where the cross point happens at $\left(a_{0}, b_{0}\right)=(0,0)$. Also, instead of the geometrical quantities $R, a, k, \delta$ defined above, we are going to use the curve vertices coordinates $\left(a_{i}, b_{i}\right), i=1,2,3$, (see Figure 1, right) in order to describe the main features of the curve. In particular, this means that the tangent lines at points $\left(a_{1}, b_{1}\right)$ and $\left(a_{3}, b_{3}\right)$ are vertical and the tangent line at point $\left(a_{2}, b_{2}\right)$ is horizontal.

It is easy to relate these coordinates with the previous parameters. For instance:

$$
a_{1}=R+a, a_{2}=2 R-2 a \delta, a_{3}=R-a, b_{2}=2 a k .
$$

(in the case of $\left(a_{0}, b_{0}\right) \neq(0,0)$, we would have the relation $\delta=\left(2 R-a_{2}-a_{0}\right) /(2 a)$ and $2 b=b_{2}-b_{0}$ instead of the second and the fourth previous ones). We remark that as we are dealing with curves with a cross point, additional parameters may be used to define such a curve as, for instance, the slopes $m_{0}, m_{1}$ of the branches of the curve at this cross point.

To see how the methods we propose work, we are going to apply them to different sample problems, that we describe below. Notice that other combinations of the constraints we are going to give are also possible.

\subsection{The balloon problem with a right corner (and fixed slopes)}

In this problem we impose the following constraints (see Figure 1, left): 
1) $(0,0)$ is a cross point of the curve.

2) The curve is tangent at the vertical lines $x=a_{1}$ and $x=a_{3}$, and also to the horizontal one $y=b_{2}$.

3) We fix $m_{0}, m_{1}$, the right and left slopes at $(0,0)$, respectively. We may have a right angle condition, that is $m_{0} \cdot m_{1}=-1$.

\subsection{The F4E problem}

In this problem we impose the following constraints (see Figure 1, middle):

1) $(0,0)$ is a cross point of the curve.

2) The curve passes through $\left(a_{1}, b_{1}\right)$ and $\left(a_{3}, b_{3}\right)$. We may or may not impose $b_{1}=b_{3}$.

3) Vertical tangencies at the previous points.

4) We fix $m_{0}, m_{1}$, the right and left slopes at $(0,0)$, respectively. We may have a right angle condition, that is $m_{0} \cdot m_{1}=-1$.

\section{The F4E problem with fixed height}

In this problem we impose the same constraints as in the F4E problem 2.2, but also the following one:

5) the curve has horizontal tangent $y=b_{2}$.

\section{The F4E problem with fixed top and height}

In this problem we impose the same constraints as in the F4E problem 2.2, but also the following one (see Figure 1, right):

6) the curve has horizontal tangent $y=b_{2}$ at the point $\left(a_{2}, b_{2}\right)$.

\section{Method 1: polynomial interpolation}

In this section we obtain solutions of the problem by parametric polynomial interpolation, that is, we are going to look for curves of the form

$$
(x(t), y(t))=\left(x_{0}+x_{1} t+x_{2} t^{2}+\cdots+x_{m} t^{m}, y_{0}+y_{1} t+y_{2} t^{2}+\cdots+y_{n} t^{n}\right), \quad t \in[0,1]
$$

whose coefficients will be determined by the constraints of the problem. Observe that the degrees of the polynomials $m, n$ will depend on the amount of constraints we impose on the definition of the curve.

As we are going to see, the constraints we will be dealing with can be written as linear equations on the previous polynomial coefficients. Hence, finding the polynomials will simply consist of finding the solution of a linear system of equations.

First of all, and without loss of generality, we are going to assume that the cross point $(0,0)$ is achieved at $t=0$ and $t=1$. Therefore, we immediately obtain that

$$
x_{0}=y_{0}=0 \text {, }
$$




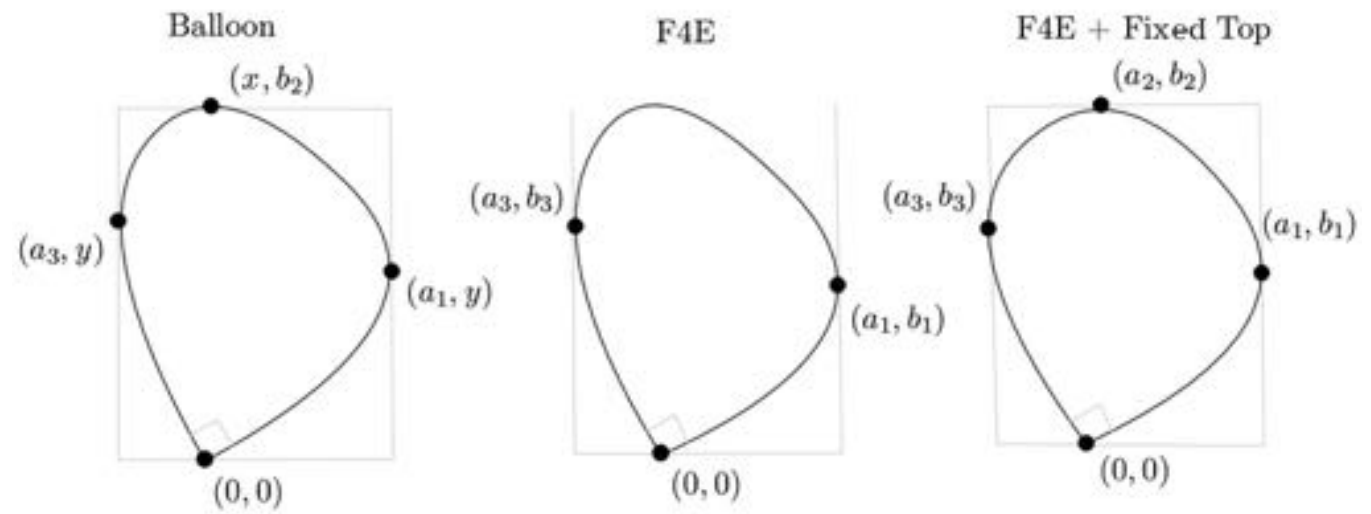

Figure 1: Diagrams of three different example problems. From left to right, the balloon problem with a right corner, the $\mathrm{F} 4 \mathrm{E}$ problem and the $\mathrm{F} 4 \mathrm{E}$ with a fixed top problem.

$$
\begin{aligned}
x_{1}+\ldots+x_{m} & =0, \\
y_{1}+\ldots+y_{n} & =0 .
\end{aligned}
$$

Apart from the previous cross point condition, the constraints on the vertices conditions at points $\left(a_{i}, b_{i}\right), i=1,2,3$, will be imposed at times $t_{1}, t_{2}, t_{3}$, respectively. This times can be specified by the user. Actually, we can obtain many interpolating curves by using $t_{i}$ as tunning parameters to fit the target curve.

The constraints on the vertices (and, hence, the corresponding linear equations on the coefficients) are from the following three types:

(i) the curve has to pass through a given point $\left(a_{i}, b_{i}\right)$ at a given time $t=t_{i}$, that is, $\left(x\left(t_{i}\right), y\left(t_{i}\right)\right)=\left(a_{i}, b_{i}\right)$. Imposing this condition leads to the following linear equations for the coeficients $x_{i}, y_{j}$ :

$$
\begin{aligned}
x_{1} t_{i}+x_{2} t_{i}^{2}+\cdots+x_{m} t_{i}^{m} & =a_{i}, \\
y_{1} t_{i}+y_{2} t_{i}^{2}+\cdots+y_{n} t_{i}^{n} & =b_{i} .
\end{aligned}
$$

(ii) The curve has to be tangent to the vertical line $x=a_{i}$ or the horizontal one $y=b_{i}$ at $t=t_{i}$. Imposing these conditions, we obtain $x\left(t_{i}\right)=a_{i}$ and $x^{\prime}\left(t_{i}\right)=0$ in the first case, that is:

$$
\begin{aligned}
x_{1} t_{i}+x_{2} t_{i}^{2}+\cdots+x_{m} t_{i}^{m} & =a_{i}, \\
x_{1}+2 x_{2} t_{i}+\cdots+m x_{m} t_{i}^{m-1} & =0
\end{aligned}
$$

or $y\left(t_{i}\right)=b_{i}$ and $y^{\prime}\left(t_{i}\right)=0$ in the second one, that is

$$
\begin{aligned}
y_{1} t_{i}+y_{2} t_{i}^{2}+\cdots+y_{n} t_{i}^{n} & =b_{i}, \\
y_{1}+2 y_{2} t_{i}+\cdots+n y_{n} t_{i}^{n-1} & =0 .
\end{aligned}
$$

In the case of having a tangency condition exactly at the point $\left(a_{i}, b_{i}\right)$, we would combine (1) with the corresponding previous conditions on the derivatives. 
(iii) Slopes or angle condition. We recall that the slope of the curve at a certain point $(x(t), y(t))$ is simply $y^{\prime}(t) / x^{\prime}(t)$. In particular, for $t=0$ and $t=1$, the slopes $m_{0}, m_{1}$ satisfy the equations

$$
m_{0} x_{1}-y_{1}=0
$$

and

$$
m_{1}\left(x_{1}+2 x_{2}+\cdots+m x_{m}\right)-\left(y_{1}+2 y_{2}+\cdots+n y_{n}\right)=0,
$$

respectively. Observe also that the angle at $(0,0)$ will be of 90 degrees if $m_{0} \cdot m_{1}=$ -1 . More generally, an angle $\alpha_{0}$ at $(0,0)$ would be obtained by imposing the condition:

$$
\cos \alpha_{0}=\frac{-1-m_{0} m_{1}}{\sqrt{\left(1+m_{0}^{2}\right)\left(1+m_{1}^{2}\right)}} .
$$

Remark 3.1. It is worth to say that other types of interpolation can be used (such as splines), that may be also convenient from a numerical point of view. We have to say that in this case we would obtain a piecewise parametrization.

Also, when the only conditions are those requiring that the curve passes through a set of given points, there are well-known ways and methods to obtain the interpolating polynomial more efficiently.

To illustrate the method, we are going to state and solve different problems, that have already been described in Section 2.

\subsection{The balloon problem with a right corner (and fixed slopes)}

This problem is described in 2.1 of Section 2, but we add here the times $t_{i}$ where the corresponding constraints must be fulfilled.

1) $(0,0)$ is a cross point and the curve passes through it at $t=0$ and $t=1$.

2) Tangency conditions at $x=a_{1}, y=b_{2}, x=a_{3}$ at times $t_{1}, t_{2}, t_{3}$ respectively.

3) Fixed slopes $m_{0}, m_{1}$ at $(0,0)$ when $t=0$ and $t=1$. We may have a right angle condition, that is $m_{0} \cdot m_{1}=-1$.

In this case, $(x(t), y(t))$ can be obtained as polynomials both of degree 5 if we include the slope conditions as conditions to determine the coefficients of $y(t)$. In the second plot of Figure 2 we can see an example of this problem solve by method 1 .

\subsection{The F4E problem}

This problem is described in 2.2 of Section 2, but we add here the times $t_{i}$ where the corresponding constraints must be fulfilled. In this problem we impose the following constraints:

1) $(0,0)$ is a cross point and the curve passes through it at $t=0$ and $t=1$.

2) The curve passes through $\left(a_{1}, b_{1}\right)$ and $\left(a_{3}, b_{3}\right)$ at $t=t_{1}, t=t_{3}$ respectively. We may or may not impose $b_{1}=b_{3}$.

3) Vertical tangencies at the previous points at $t=t_{1}, t=t_{3}$ respectively. 
4) Fixed slopes $m_{0}, m_{1}$ at $(0,0)$ when $t=0$ and $t=1$. We may have a right angle condition, that is $m_{0} \cdot m_{1}=-1$.

In this case, $(x(t), y(t))$ can be obtained as polynomials both of degree 5 if we include the slope conditions as conditions to determine the coefficients of $y(t)$. In the third plot of Figure 2 we can see an example of this problem.

\section{The F4E problem with fixed top and height}

This problem is described in 2.2 of Section 2. The times times $t_{i}$ where the corresponding constraints must be fulfilled are the previous ones for the F4E problem, and also

$5)$ the curve passes through $\left(a_{2}, b_{2}\right)$ and has horizontal tangent at this point at $t=t_{2}$.

In this case, $(x(t), y(t))$ can be obtained as polynomials of degree 6 and 7 , respectively, if we include the slope conditions as conditions to determine the coefficients of $y(t)$. See Figure 2, right, for an example of this problem.
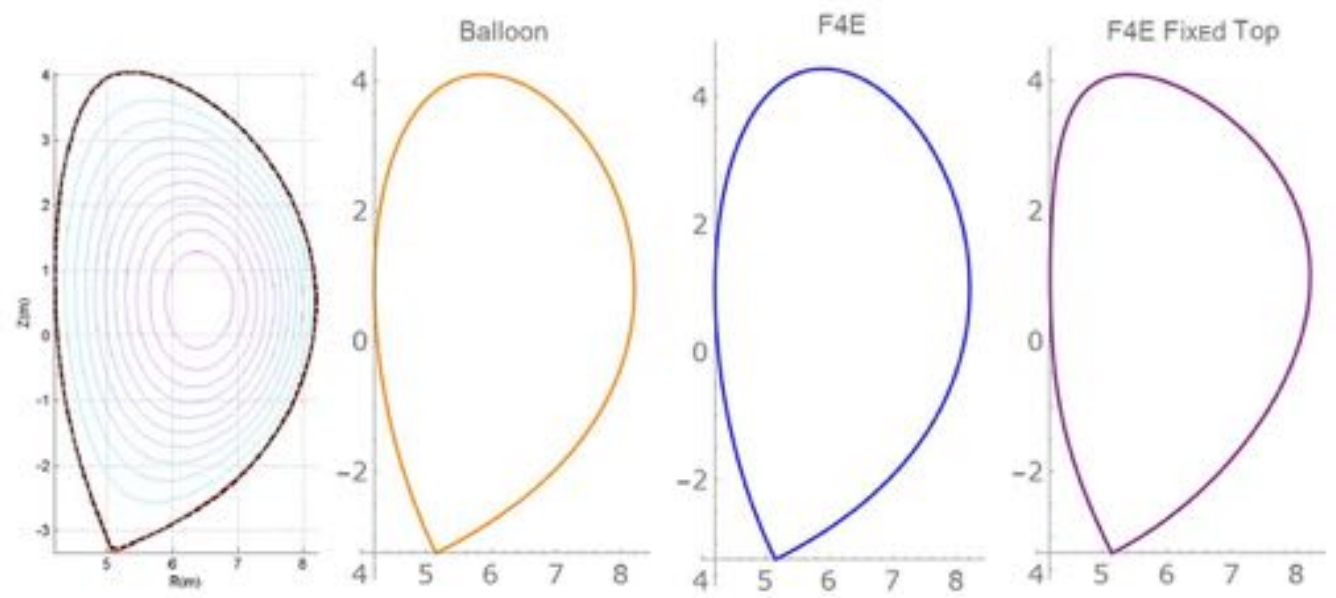

Figure 2: Simulation of method 1 (polynomial interpolation) for the three different problems described above (second, third and fourth plots). Compare with the left figure which is a representation of the plasma given in [3].

\section{Method 2: level curves}

Motivated by the level curves of a Hamiltonian with a cubic potential energy, our approach in this section will consist of finding an implicit representation of the curve as the zero level set of a cubic Hamiltonian. That is, finding a cubic function, whose general form is

$H(x, y)=\alpha_{00}+\alpha_{10} x+\alpha_{01} y+\alpha_{20} x^{2}+\alpha_{11} x y+\alpha_{02} y^{2}+\alpha_{30} x^{3}+\alpha_{21} x^{2} y+\alpha_{12} x y^{2}+\alpha_{03} y^{3}$ such that its zero level curve $\left(\Gamma_{0}=\left\{(x, y) \in \mathbb{R}^{2}, H(x, y)=0\right\}\right)$ fits the target conditions. 
Using this approach we would also obtain the set of level curves $\Gamma_{c}=\{(x, y) \in$ $\left.\mathbb{R}^{2}, H(x, y)=c\right\}$ and that these curves would be generically regular when $c \neq 0$.

Observe that as $(0,0)$ must be a saddle point, we immediately obtain that $\alpha_{00}=\alpha_{10}=$ $\alpha_{01}=0\left(\right.$ as $\left.H(0,0)=H_{x}(0,0)=H_{y}(0,0)=0\right)$. Observe also that we can then assume $\alpha_{20} \neq 0$ without loss of generality to avoid that the vertical axis is one of the saddle axis. So, we can divide all the coefficients by $\alpha_{20}$ and, hence, the cubic we are looking for has the general form

$$
H(x, y)=x^{2}+\alpha_{11} x y+\alpha_{02} y^{2}+\alpha_{30} x^{3}+\alpha_{21} x^{2} y+\alpha_{12} x y^{2}+\alpha_{03} y^{3} .
$$

In this curve we have six coefficients to be fixed. This will be done imposing up to six constraints on the problem. A natural way to fix all of them is to impose that the vertices of the curve are $\left(a_{i}, b_{i}\right), i=1,2,3$. However, in general we will not obtain a right angle at $(0,0)$. A way of getting it could be not fixing one of the vertices (for instance, $\left(a_{2}, b_{2}\right)$ ) and use it as a tunning parameter to achieve the right angle condition, as we will see below.

Although the curve (2) is given in an implicit form, there exists a classical method to obtain its parametrization. Consider the sheaf of straight lines passing through the origin $(0,0)$, that is, $y=t x$. Observe that $t$ has a geometrical meaning: it represents the slope with respect to the horizontal axis at the point $(0,0)$. The method consists in observing that each of these lines intersects at three points with the cubic. But, as $(0,0)$ is a cross point of the curve, two of these points are $(0,0)$, so the third intersection point gives us a parametrization of the curve:

$$
x(t)=-\left(\frac{\alpha_{02} t^{2}+\alpha_{11} t+1}{\alpha_{03} t^{3}+\alpha_{12} t^{2}+\alpha_{21} t+\alpha_{30}}\right), \quad y(t)=t x(t)
$$

with $t \in\left[m_{1}, m_{0}\right]$, where $m_{0}, m_{1}$ stand for the right and left slopes at $(0,0)$ with respect to the horizontal axis, respectively:

$$
m_{0}=\frac{2}{-\alpha_{11}+\sqrt{\alpha_{11}^{2}-4 \alpha_{02}}}, m_{1}=\frac{2}{-\alpha_{11}-\sqrt{\alpha_{11}^{2}-4 \alpha_{02}}} .
$$

Notice that

$$
\alpha_{11}=-\left(\frac{m_{0}+m_{1}}{m_{0} m_{1}}\right), \alpha_{02}=\frac{1}{m_{0} m_{1}} .
$$

The right angle case would be when $m_{0} \cdot m_{1}=-1$, that is, $\alpha_{02}=-1$.

The type of constraints we may have are the following:

(i) the curve has to pass through a given point $\left(a_{i}, b_{i}\right)$, that is:

$$
H\left(a_{i}, b_{i}\right)=0
$$

which is a linear equation on the coefficients $\alpha_{i j}$.

(ii) Tangency conditions. Observe that if the tangency conditions happen at a particular point $\left(a_{i}, b_{i}\right)$, then we have

$$
H\left(a_{i}, b_{i}\right)=H_{y}\left(a_{i}, b_{i}\right)=0
$$

for a vertical tangent or

$$
H\left(a_{i}, b_{i}\right)=H_{x}\left(a_{i}, b_{i}\right)=0
$$

for an horizontal one, which are linear equations on the coefficients. 
If the curve has to be tangent to the vertical line $x=a_{i}$ or the horizontal one $y=b_{i}$, it must be fulfilled that

$$
H\left(a_{i}, y\right)=H_{y}\left(a_{i}, y\right)=0 \text { or } H\left(x, b_{i}\right)=H_{x}\left(x, b_{i}\right)=0,
$$

respectively. But observe that in these equations there are $x$ or $y$ as new unknowns (if we knew the exact points where these tangencies occur, then these would be linear equations on the coefficients). Hence, a better way to think these generic vertical or horizontal tangencies is through the discriminant of the cubic. More concretely, if the curve has to be tangent to the vertical line $x=a_{i}$, this would happen at a double root of the $y$-cubic function $H\left(a_{i}, y\right)$. So, $H\left(a_{i}, y\right)$ would be a cubic $y$-polynomial with zero discriminant (to assure this double root happen). Hence, the conditions (5) could be replaced by the corresponding zero discriminant conditions, which are nonlinear equations of the coefficients.

(iii) Slope conditions. We have seen in (3) the relation between the slopes $m_{0}, m_{1}$ at $(0,0)$ in terms of the coefficients of the cubic function $H$. Recall that the case of a right angle at $(0,0)$ corresponds to $\alpha_{02}=-1$. In the case of imposing given slopes, the coefficients $\alpha_{11}$ and $\alpha_{02}$ are fixed by (4).

As we have said, in the general form (2) of the cubic we are looking for there are six coefficients to be fixed. So, to fix all of them we would need exactly six appropriate conditions on the curve. In other words, in problems with less constraints (such as, for instance, the balloon problem with a right corner or the F4E problem) we will obtain a cubic curve of the form (2) but with some free coefficients. These coefficients can be turned into zero or we can use them as tunning parameters to achieve the desired curve.

To illustrate this method, we are going to apply it to the F4E problem described in Section 2 and see how the free parameters can be used to fit the target curve.

\subsection{The F4E problem}

Following the requirements of the F4E problem (see 2.2 in Section 2), the first condition to be achieved is a right angle at $(0,0)$. This condition yields to

$$
H(x, y)=x^{2}+\alpha_{11} x y-y^{2}+\alpha_{30} x^{3}+\alpha_{21} x^{2} y+\alpha_{12} x y^{2}+\alpha_{03} y^{3} .
$$

Fixing the two lateral vertices at $\left(a_{1}, b_{1}\right)$ and $\left(a_{3}, b_{3}\right)$ corresponds to four linear equations of the remaining coefficients. Hence, we will have a free parameter, that can be chosen to be, for instance, $\alpha_{30}$. This means that we can choose it either to be zero or we can use it as a tunning parameter to achieve the target curve.

\section{The F4E problem with fixed height}

Following the previous approach, we can adjust $\alpha_{30}$ in order to get a desired height $b_{2}$, that is, to get an horizontal tangency at $y=b_{2}$. In Figure 3 (right) we can see the relation between this $\alpha_{30}$ and $b_{2}$. A simulation of this second method for a F4E problem can be seen in Figure 3 (middle).

Another point of view for solving the F4E problem with fixed height is imposing the tangency condition at $y=b_{2}$ as a discriminant condition, getting an extra cubic equation for $\alpha_{30}$. 

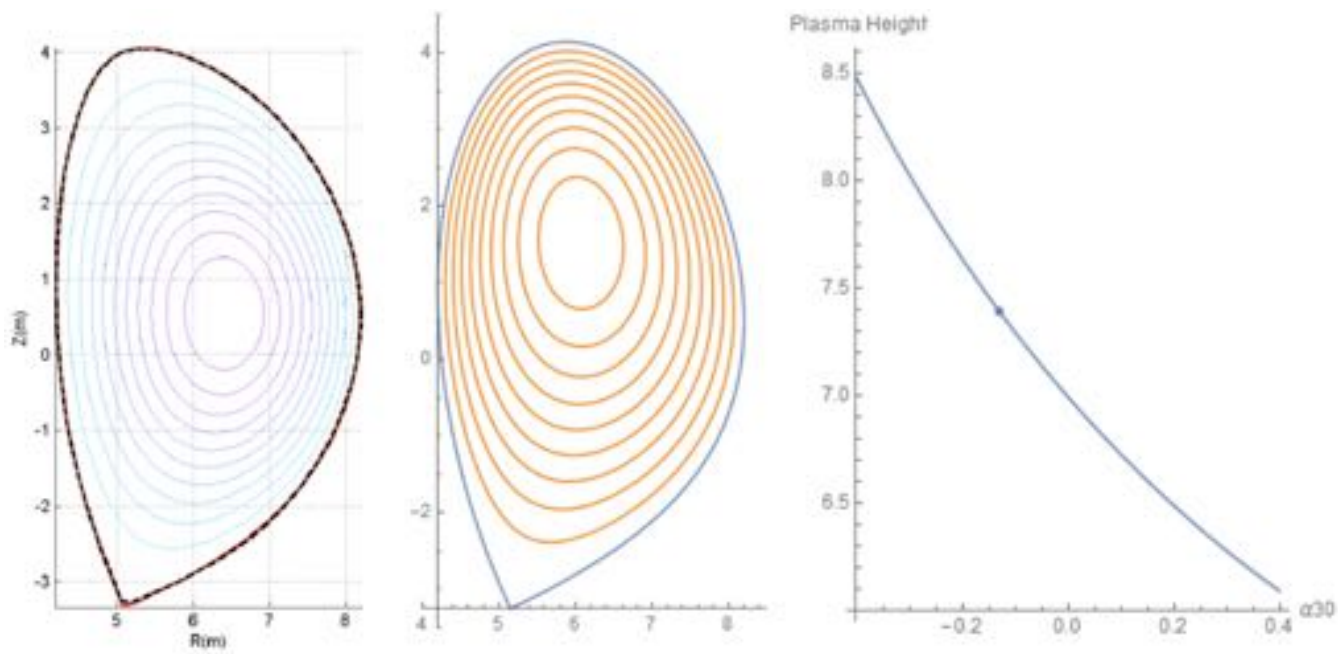

Figure 3: In the middle, simulation of method 2 applied to the F4E problem. See also the different level curves of the cubic Hamiltonian and compare with the left figure which is a representation of the plasma given in [3]. The right curve shows the relation between the plasma height $b_{2}$ and the free parameter $\alpha_{30}$ (the point corresponds to the value used in the simulation, that has been chosen to fit the desired height of the plasma, according to its representation given in left figure).

In conclusion, using an implicit cubic representation of the curve allows a maximum of six constraints. If we want to impose more constraints, we should use a higher order implicit polynomial representation. For instance, an implicit quartic representation would add the possibility of five more free parameters to be adjusted. Notice, however, that in this case we generally do not have a rational parametric representation of the curve but an irrational one.

\section{Some ideas for Problem 2}

In Problem 2 presented by F4E, one is asked to find the currents along the exterior coils to produce a scalar field $\Psi$ on the boundary $\Gamma$ of the plasma region that matches a given target function. This target scalar field is supposed to be previously found as a solution of a Grad-Shafranov equation in a fixed domain (see subsection ?? for details).

Though important contributions were made in [3], the problem seems to be quite involved, but it has many of the characteristics of the next problem, that we suggest because it is simpler and perhaps suitable for a mathematical warm-up in the stated direction.

Let $\Omega \subset \mathbb{R}^{2}$ be an open set with a simple boundary $\Gamma$. It is known (for example, see [1] for details) that every harmonic function $u(\vec{x})$ in $\Omega$ that is continuous up to $\Gamma$ can be represented as the resultant of a continuous distribution of double layer dipoles oriented 
in the direction of the exterior normals to $\Gamma$ and with intensities $\mu(\vec{\xi})$, that is

$$
u(\vec{x})=-\frac{1}{2 \pi} \int_{\Gamma} \mu(\vec{\xi}) \frac{\partial}{\partial \vec{\nu}_{\vec{\xi}}} \ln |\vec{\xi}-\vec{x}| d \ell_{\vec{\xi}} .
$$

To get some intuition, one can consider the case where $\Gamma$ is a circle centered at $(0,0)$. In this case,

$$
\frac{\partial}{\partial \vec{\nu}_{\vec{\xi}}} \ln |\vec{\xi}-\vec{x}|=\frac{\vec{\xi} \cdot(\vec{\xi}-\vec{x})}{|\vec{\xi}-\vec{x}|^{3}} .
$$

It is known that the density function $\mu(\vec{x})$ is the solution of the following Fredholm integral equation of the second kind:

$$
\mu(\vec{x}(s))-\frac{1}{\pi} \int_{\Gamma} \mu(\vec{x}(t)) \frac{\partial}{\partial \vec{\nu}_{\vec{\xi}}} \ln |\vec{\xi}(s)-\vec{x}(t)| d t=-2 u(\vec{x}(s)),
$$

where $s$ and $t$ are counterclockwise arc-length coordinates along $\Gamma$.

Let now $\Omega_{1} \subset \Omega_{2} \subset \mathbb{R}^{2}$ be two open sets with simple boundaries $\Gamma_{1}$ and $\Gamma_{2}$ respectively. What it is not true is that every such function $u(\vec{x})$ defined in $\Omega_{1}$ can be represented as a continuously distributed double-layer potential along $\Gamma_{2}$. This can hold in some particular cases, but in general it is an ill-posed problem. To see if this function $u(\vec{x})$ coincides with the function $v(\vec{x})$ defined by a double-layer potential distribution along $\Gamma_{2}$ it would be enough to see that they coincide on $\Gamma_{1}$.

Even worse is the discretized problem, where the dipoles are a finite number $n$ of point-dipoles distributed along $\Gamma_{2}$, namely at the points $\vec{\xi}_{1}, \vec{\xi}_{2} \ldots \vec{\xi}_{n} \in \Gamma_{2}$ with intensities (moments) $\mu_{1}, \mu_{2} \ldots \mu_{n}$ (directed along the unit exterior normals of $\Gamma_{2}$ ), and the integral is substituted by a finite sum.

But even if this problem has in general no solution, we can ask ourselves to minimize the error when approximating in such a way a function $u(\vec{x})$ in $\Omega_{1}$, or more precisely on $\Gamma_{1}$ :

Problem A. Given $m$ points $\vec{x}_{1}, \vec{x}_{2} \ldots \vec{x}_{m}$ in $\Gamma_{1}$ and $n$ (fixed) points $\vec{\xi}_{1}, \vec{\xi}_{2} \ldots \vec{\xi}_{n} \in \Gamma_{2}$, find the intensities $\mu_{1}, \mu_{2} \ldots \mu_{n}$ that minimize

$$
\sum_{i=1}^{m}\left(v\left(x_{i}\right)-u\left(x_{i}\right)\right)^{2}
$$

where

$$
v(\vec{x})=\sum_{j=1}^{n} \mu_{j} \frac{\partial}{\partial \vec{\nu}_{\vec{\xi}}} \ln \left|\vec{\xi}_{j}-\vec{x}\right|
$$

Problem B. Same problem as before, but letting the locations $\vec{\xi}_{1}, \vec{\xi}_{2} \ldots \vec{\xi}_{n} \in \Gamma_{2}$ to be also variable.

\section{Acknowledgements}

The authors thank Alfredo Portone and the European Agency Fusion for Energy for proposing this problem to the 115th European Study Groups with Industry. The authors would also like to thank Jesús Fernández and Giorgi Khimshiashvili for their helpful discussions on the problem. 
This work is partially supported by the Spanish grants MTM2014-52402-C3-1-P (J. Solà-Morales), MTM2014-52402-C3-2-P (C. Barril) and MTM2014-52402-C3-3-P (M. Pellicer). Also, C. Barril, M. Pellicer and J. Solà-Morales are part of the Catalan research group 2014-SGR-1083, and M. Pellicer is also partially supported by MPC UdG 2016/047 (U. de Girona, Catalonia). À. Haro acknowledges financial support from the Spanish grant MTM2015-67724-P and the Catalan grant 2014-SGR-1145.

\section{Bibliography}

[1] A.V. Bitsadze. Equations of Mathematical Physics. Mir Publishers, Moscou (1980).

[2] R.L. Miller, M.S. Chu, J.M. Greene, Y.R. Lin-Liu, and R.E. Waltz. Noncircular, finite aspect ratio, local equilibrium model. Physics of Plasmas 5 (1998), 973-978.

[3] A. Portone. Magnet system optimization in tokamak engineering. Decision and Control (CDC), 2012 IEEE 51st Annual Conference on (4328-4334). IEEE.

[4] T. Takeda and S. Tokuda. Computation of MHD Equilibrium of Tokamak Plasma. J. Comput. Phys. 93 (1991), 1-107. 